\title{
Prevalence and Pattern of Soil-Transmitted Helminthic Infection among Primary School Children in a Rural Community in Imo State, Nigeria
}

\author{
Kelechi Kenneth Odinaka, ${ }^{1}$ Emeka Charles Nwolisa, ${ }^{2}$ Francis Mbanefo, ${ }^{2}$ \\ Alfreda Chinekwu Iheakaram, ${ }^{2}$ and Seline Okolo ${ }^{3}$ \\ ${ }^{1}$ Department of Paediatrics, Imo State University Teaching Hospital, P.O. Box 1644, Orlu, Imo State, Nigeria \\ ${ }^{2}$ Department of Paediatrics, Federal Medical Centre, Owerri, Imo State, Nigeria \\ ${ }^{3}$ Department of Paediatrics, University of Jos Teaching Hospital, Plateau State, Nigeria \\ Correspondence should be addressed to Kelechi Kenneth Odinaka; kellymed112@yahoo.com
}

Received 26 June 2015; Accepted 31 August 2015

Academic Editor: Kenneth E. Olson

Copyright ( 2015 Kelechi Kenneth Odinaka et al. This is an open access article distributed under the Creative Commons Attribution License, which permits unrestricted use, distribution, and reproduction in any medium, provided the original work is properly cited.

\begin{abstract}
Background. Soil-transmitted helminthic infection is a common public health challenge of primary school children in resource challenged and developing countries. Our aim was to determine the prevalence and pattern of soil-transmitted helminthic infection among primary school children in a rural community in Imo State, Nigeria. Method. The study involved a cross-sectional survey of 284 primary school children in a rural community. Results. The overall prevalence of soil-transmitted helminthic infection (STHI) was $30.3 \%$. Of all STHIs, hookworm was the commonest geohelminth observed, 81 (94.2\%). The prevalence of soil-transmitted helminthic infection was more in males, 58 (38.4\%), than in females, $28(21.1 \%)$. This difference was statistically significant $(P=$ 0.001). Conclusion. The high prevalence rate of soil-transmitted helminthic infection amongst the study population is worrisome. There is need for organized and routine deworming among school children in the community.
\end{abstract}

\section{Introduction}

Soil-transmitted helminths thrive and persist in human communities in which poverty, inadequate sanitation, lack of access to health care, and overcrowding are entrenched [1]. Additionally, the habits of bare feet on sand and eating unwashed fruits and vegetables also encourage the transmission of helminthic infection [2]. These habits occur in most rural communities and urban slums in resource challenged and developing countries including Nigeria. The World Health Organization (WHO) estimates that over two billion people are infected with one or more soil-transmitted helminths, mainly Ascaris lumbricoides, hookworm, and Trichuris trichiura [3]. School-aged children have been shown to be the population at greatest risk of acquiring infections with roundworm, hookworm, and whipworm infections [1, 4]. The preponderance of helminthic infection in school-aged children makes this subgroup a good target for helminth control programmes in the general population and schools provide good opportunities for implementation of control programmes $[5,6]$. Intestinal helminthiasis is often associated with reduced physical activity and may worsen the already compromised nutritional status of the school-aged children in rural communities.

The prevalence of soil-transmitted helminthiasis differs from region to region. Although several studies $[7,8]$ have been conducted on the prevalence of intestinal helminthiasis in Nigeria, there are still localities including the study area for which epidemiological information is not available. WHO recommends that a baseline survey in school children be done to determine prevalence of worm infestation before instituting any worm control programme and treatment should be given according to the survey [9]. Presently, there is no national school-based helminth control programme in Nigeria. In the past, there have been sporadic and uncoordinated deworming programmes instituted by politicians 
and philanthropists. This study sought to determine the prevalence and pattern of soil-transmitted helminthiasis. This study will serve as a baseline for any future evaluation of proposed regular deworming programmes at schools in the area and the state in general.

\section{Method}

2.1. Study Area. The study was conducted in Amaruru, Orsu Local Government Area (LGA) of Imo State in Southeastern Nigeria. The community lies within the tropical rain forest zone of Africa and has the characteristic wet and dry seasons. Amaruru is predominantly an agrarian community with a mixture of traders, artisans, and civil servants. There are three government approved primary schools in Amaruru. None of the schools had water supply or toilet facility.

2.2. Study Design. The study was a cross-sectional, schoolbased, descriptive study. The participants for the study were selected using a systematic sampling technique. The study was performed between September and November 2012.

2.3. Study Population. The study population comprised children from primary one through primary six in all the three schools. The age of the children was determined from the school record.

2.4. Ethical Approval. Approval to carry out the study was obtained from the Ethics Committee of the Federal Medical Centre, Owerri, and Imo State Universal Basic Education Board. Written informed consent was obtained from parents/guardians of the subjects through the Parents-Teachers Association. Assent was also obtained from the children.

2.5. Laboratory Methods. Stool samples were examined using Stoll's dilution technique [10]. A small sized, clean, dry, and leakproof plastic container with a wide mouth and prelabelled with the subject's name and an identification number was issued to each recruited child. Students with the help of parents or guardians were instructed to bring to school stool specimens collected that morning. The stool sample was taken to the Federal Medical Centre, Owerri, parasitology laboratory within six hours after the stool had been passed, to be analysed for the presence of ova of soil-transmitted helminths. The pupils submitting stool samples were given pencils, erasers, or pens as incentives. All infected children were given a single dose of mebendazole $(500 \mathrm{mg})$ at the expense of the researcher. Social classification of the children was based on the criteria set by Oyedeji (1985) [11].

2.6. Data Management and Analysis. Statistical analysis of the data was performed using the Statistical Package for the Social Sciences (SPSS) for Windows (version 16.0). Comparative analysis involving two categorical variables was done using chi-square test. The level of significance of each test was set at $P<0.05$.
TABLE 1: Sociodemographic characteristics of the study population.

\begin{tabular}{lcc}
\hline Characteristic & $\begin{array}{c}\text { Frequency } \\
(n)\end{array}$ & $\begin{array}{c}\text { Percentage } \\
(\%)\end{array}$ \\
\hline Age group (years) & & \\
$5-7$ & 34 & 12.0 \\
$8-10$ & 112 & 39.4 \\
$11-13$ & 121 & 42.6 \\
$14-16$ & 17 & 6.0 \\
Gender & & \\
Male & 151 & 53.2 \\
Female & 133 & 46.8 \\
Socioeconomic status & & \\
Upper class & 2 & 0.7 \\
Middle class & 87 & 30.6 \\
Lower class & 195 & 68.7 \\
\hline
\end{tabular}

\section{Results}

A total of 288 children were recruited for the study; of these, $284(98.6 \%)$ submitted stool samples while $4(1.4 \%)$ did not submit stool sample. Data from these 284 subjects were analysed. The distribution of the subjects based on age group, gender, and socioeconomic class is shown in Table 1.

Of the 284 pupils studied, 86 (30.3\%) had helminth ova detected in stool. The male subjects, $58(38.4 \%)$, were more infected than the female subjects, 28 (21.1\%). This difference was statistically significant $(P=0.001)$. Pupils who never washed their hands after defecation had the highest prevalence of helminthiasis (33.3\%), followed by those who occasionally washed their hands (31.1\%), while pupils who always washed their hands had the lowest prevalence (27.1\%). The difference among the three groups in terms of personal hygiene was not statistically significant $(P=0.694)$. The data are shown in Table 2.

The prevalence of soil-transmitted helminthiasis was highest among subjects who sourced drinking water from the stream (43\%), followed by those sourcing their drinking water from boreholes (38.4\%). Subjects who sourced drinking water from springs had the lowest prevalence rate (1.2\%). Among those infected, hookworm infection had a prevalence of (94.2\%), followed by Ascaris lumbricoides (2.3\%). Trichuris trichiura was not identified in the stool of study subjects. Infections with hookworm and Ascaris lumbricoides occurred in $3.5 \%$ of the subjects (Table 3 ).

\section{Discussion}

The prevalence of soil-transmitted helminthiasis in the study population was $30.3 \%$. This classifies the community as a "moderate risk area" for preventive therapy by WHO standards [9]. Soil-transmitted helminthic infection is therefore a problem amongst primary school children aged 5-16 years in Amaruru community, in Imo State. This is similar to findings of other studies $[12,13]$ in other parts of Nigeria which also found helminthiasis to be common among primary 
TABLE 2: Risk factors and prevalence of soil-transmitted helminthiasis among the study subjects.

\begin{tabular}{|c|c|c|c|c|c|}
\hline \multirow{2}{*}{ Risk factors } & \multicolumn{2}{|c|}{ Helminth status } & \multirow[b]{2}{*}{ Total $n(\%)$} & \multirow{2}{*}{$\chi^{2}$} & \multirow{2}{*}{$P$ value } \\
\hline & Infected $n(\%)$ & Noninfected $n(\%)$ & & & \\
\hline \multicolumn{6}{|l|}{ Gender } \\
\hline Male & $58(38.4)$ & $93(61.6)$ & $151(100)$ & \multirow{3}{*}{10.09} & \multirow{3}{*}{$0.001^{*}$} \\
\hline Female & $28(21.1)$ & $105(78.9)$ & $133(100)$ & & \\
\hline Total & $86(30.3)$ & $196(69.3)$ & $284(100)$ & & \\
\hline \multicolumn{6}{|c|}{ Wearing of footwear } \\
\hline Yes & $66(26.0)$ & $188(74.0)$ & $254(100)$ & \multirow{3}{*}{0.15} & \multirow{3}{*}{0.700} \\
\hline No & $20(66.7)$ & $10(33.3)$ & $30(100)$ & & \\
\hline Total & $86(30.3)$ & $198(69.3)$ & $284(100)$ & & \\
\hline \multicolumn{6}{|c|}{ Hand washing after defecation } \\
\hline Always & $16(27.1)$ & $43(72.9)$ & $59(100)$ & \multirow{4}{*}{0.91} & \multirow{4}{*}{0.694} \\
\hline Occasionally & $69(31.1)$ & $153(69.8)$ & $222(100)$ & & \\
\hline Never & $1(33.3)$ & $2(66.7)$ & $3(100)$ & & \\
\hline Total & $86(30.3)$ & $198(69.3)$ & $284(100)$ & & \\
\hline \multicolumn{6}{|l|}{ Sewage disposal } \\
\hline Water closet & $1(6.3)$ & $15(93.7)$ & $16(100)$ & \multirow{4}{*}{4.79} & \multirow{4}{*}{0.09} \\
\hline Pit latrine & $70(32.3)$ & $147(67.7)$ & $217(100)$ & & \\
\hline Bush & $15(29.4)$ & $36(70.6)$ & $51(100)$ & & \\
\hline Total & $86(30.3)$ & $198(69.3)$ & $284(100)$ & & \\
\hline \multicolumn{6}{|c|}{ Age group (years) } \\
\hline $5-7$ & $8(23.5)$ & $26(76.5)$ & $34(100)$ & \multirow{5}{*}{4.27} & \multirow{5}{*}{0.23} \\
\hline $8-10$ & $38(33.9)$ & $74(66.1)$ & $112(100)$ & & \\
\hline $11-13$ & $38(31.4)$ & $83(68.6)$ & $121(100)$ & & \\
\hline $14-16$ & $2(11.8)$ & $15(88.2)$ & $17(100)$ & & \\
\hline Total & $86(30.3)$ & $198(69.7)$ & $284(100)$ & & \\
\hline
\end{tabular}

${ }^{*}$ Significant $P$ values.

TABLE 3: Soil-transmitted helminths identified among subjects.

\begin{tabular}{lcc}
\hline Species & $\begin{array}{c}\text { Frequency } \\
(n)\end{array}$ & $\begin{array}{c}\text { Percentage } \\
(\%)\end{array}$ \\
\hline $\begin{array}{l}\text { Hookworm } \\
\text { Ascaris lumbricoides }\end{array}$ & 81 & 94.2 \\
+ hookworm & 3 & 3.5 \\
Ascaris lumbricoides & 2 & 2.3 \\
Total & $\mathbf{8 6}$ & $\mathbf{1 0 0}$ \\
\hline
\end{tabular}

school children. A similar pattern of infection is likely to occur in many other communities in the state and other parts of Nigeria. The high prevalence rate of soil-transmitted helminths in the study population could be attributable to the risk factors associated with the study population, that is, nonavailability of water supply and toilet facilities in schools. Furthermore, the majority of children belong to a lower socioeconomic class. The prevalence rate in the present study is higher than the $24.6 \%$ observed by Okolo and John [14]. The difference between this study and the Okolo study could be due to the latter study's use of a less sensitive technique (wet preparation technique) for detecting parasites [15].

The age groups of $8-10$ and $11-13$ years recorded higher prevalence rates of $33.9 \%$ and $31 \%$, respectively. Children in these age groups engage in play activities in contaminated environments that could facilitate transmission of intestinal helminths. The children also tend to be less cautious of their personal hygiene because they are not old enough to understand the need for general cleanliness, unlike their counterparts in the age group of 14-16 years in which the infection rate was very low. A lower infection rate in the age group of 14-16 years may be due to the psychosocial development of the mid-adolescent, as they are more selfconscious of their personal hygiene and outward appearance to attract the opposite sex. Thus, mid-adolescents are less likely to walk around barefooted. Similar findings and reasons have been adduced by other researchers $[8,12,13]$.

Pit latrines and nearby bush were the commonly used sites of sewage disposal in the study population and in agreement with the earlier report of Adefioye et al. [8]. The use of pit latrine and nearby bush reflects the poor socioeconomic status of the study subjects. Additionally, students in the schools had no access to clean water supply and toilet facility. Soil-transmitted helminths thrive and flourish in such communities.

Our study also showed that the prevalence of soiltransmitted helminthiasis was higher in males than in females. This could be because the majority of the study population were children of farmers and males usually accompany their fathers to the farm. Male children are also 
known to be more adventurous. This is in agreement with the report of Obiukwu et al. [12].

Multiple infections occurred in 3.5\% of the subjects with confirmed soil-transmitted helminthic infection. The multiple soil-transmitted helminthic infections observed may be as a result of multiple infection risks that they are exposed to during their daily activities.

Hookworm was the most common soil-transmitted helminthic infection identified in this study and may be attributed to the rainy season when the study was conducted. The observation of increased hookworm transmission during the rainy season has been documented [16]. The plausible reason for this increased hookworm transmission during the rainy season may be that the rains disperse faeces increasing chances of parasite contact with humans. Trichuris trichiura infection was not observed similar to studies reported by Adefioye et al. [8]. Trichuris trichiura generally has a very low prevalence as documented in some studies $[17,18]$. However, the absence of Trichuris trichiura in this study may be due to the single stool examination technique utilized here. Multiple stool examinations may have improved the yield of ova of Trichuris trichiura.

\section{Conclusion}

The finding of a high prevalence of soil-transmitted helminthic infection amongst primary school children in Amaruru in Imo State, Nigeria, emphasises the need for potable water supply and safe sewage disposal systems in schools. There is also a need for routine regular deworming of all the students in the schools to reduce the burden of soiltransmitted helminthiasis and guarantee the delisting of the community's WHO “moderate risk area” classification status.

\section{Conflict of Interests}

The authors declare that there is no conflict of interests regarding the publication of this paper.

\section{References}

[1] World Health Organization, "Prevention and control of schistosomiasis and soil transmitted helminthiasis," WHO Technical Report Series 912: i-vi, World Health Organization, Geneva, Switzerland, 2002.

[2] A. Monstresor, D. W. T. Crompton, A. Bundy, A. Hall, and L. Savioli, Guidelines for the Evaluation of Soil-transmitted Helminthiasis and Schistosomiasis at Community Level, WHO/ CTD/SIP/98.1, World Health Organization, Geneva, Switzerland, 1998.

[3] J. Bethony, S. Brooker, M. Albonico et al., "Soil-transmitted helminth infections: ascariasis, trichuriasis, and hookworm," The Lancet, vol. 367, no. 9521, pp. 1521-1532, 2006.

[4] N. R. De Silva, S. Brooker, P. J. Hotez, A. Montresor, D. Engels, and L. Savioli, "Soil-transmitted helminth infections: updating the global picture," Trends in Parasitology, vol. 19, no. 12, pp. 547$551,2003$.

[5] M. C. Jukes, C. A. Nokes, K. J. Alcock et al., "Heavy schistosomiasis associated with poor short-term memory and slower reaction times in Tanzanian schoolchildren," Tropical Medicine \& International Health, vol. 7, no. 2, pp. 104-117, 2002.

[6] World Health Organization, "The evidence is in: deworming helps to meet millennium development goals," WHO Technical Report, World Health Organization, Geneva, Switzerland, 2005.

[7] J. E. Olaniyi, H. A. Muktar, and P. E. Jolly, "A review of intestinal helminthiasis in Nigeria and the need for schoolbased intervention," Journal of Rural and Tropical Public Health, vol. 6, pp. 33-39, 2007.

[8] O. A. Adefioye, A. M. Efunshile, O. Ojurongbe et al., "Intestinal helminthiasis among school children in Ilie, Osun State, Southwest, Nigeria," Sierra Leone Journal of Biomedical Research, vol. 3, no. 1, pp. 36-42, 2011.

[9] World Health Organization, Soil-Transmitted Helminthiasis: Eliminating Soil-Transmitted Helminthiasis as a Public Health Problem in Children: Progress Report 2001-2010 and Strategic Plan 2011-2020, World Health Organization, Geneva, Switzerland, 2012.

[10] M. Cheesbrough, "Techniques used to identify parasites," in Medical Laboratory Manual for Tropical Countries, M. Cheesbrough, Ed., pp. 178-193, Cambridge University Press, Cambridge, UK, 2nd edition, 1992.

[11] G. A. Oyedeji, "Socioeconomic and cultural background of hospitalized children in Ilesha," Nigerian Journal of Paediatrics, vol. 12, no. 4, pp. 111-117, 1985.

[12] M. O. Obiukwu, P. U. Umeanaeto, C. I. Eneanya, and G. O. Nwaorgu, "Prevalence of gastro-intestinal helminths in school children in Mbaukwu, Anambra State, Nigeria," Nigerian Journal of Parasitology, vol. 29, no. 1, pp. 15-19, 2008.

[13] O. M. Ukpai and C. D. Ugwu, "The prevalence of gastrointestinal tract parasites in primary school children in Ikwuano Local Government Area of Abia State Nigeria," Nigerian Journal of Parasitology, vol. 24, pp. 129-136, 2003.

[14] S. N. Okolo and C. John, "Nutritional status and intestinal parasitic infestation among rural Fulani children in Vom, Plateau State," Nigerian Journal of Paediatrics, vol. 33, no. 2, pp. 47-55, 2008.

[15] S. C. Tay, S. Y. Gbedema, and T. K. Gyampomah, "Accuracy of diagnosis of intestinal helminthic parasites in a reference diagnostic laboratory in the Ashanti region of Ghana," International Journal of Parasitology Research, vol. 3, no. 1, pp. 12-16, 2011.

[16] A. B. C. Nwosu, "The community ecology of soil-transmitted helminth infections of humans in a hyperendemic area of southern Nigeria," Annals of Tropical Medicine and Parasitology, vol. 75, no. 2, pp. 197-203, 1981.

[17] O. A. Adeyeba and B. D. Tijani, "Intestinal helminthiasis among malnourished school age children in peri-urban area of Ibadan, Nigeria," African Journal of Clinical and Experimental Microbiology, vol. 3, no. 1, pp. 24-28, 2002.

[18] F. Osazuwa, O. M. Ayo, and P. Imade, "A significant association between intestinal helminth infection and anaemia burden in children in rural communities of Edo state, Nigeria," North American Journal of Medical Sciences, vol. 3, no. 1, pp. 30-34, 2011. 


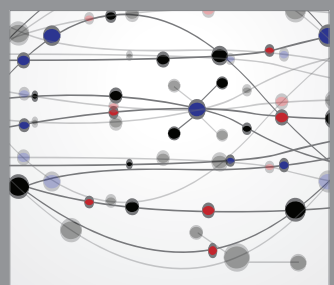

The Scientific World Journal
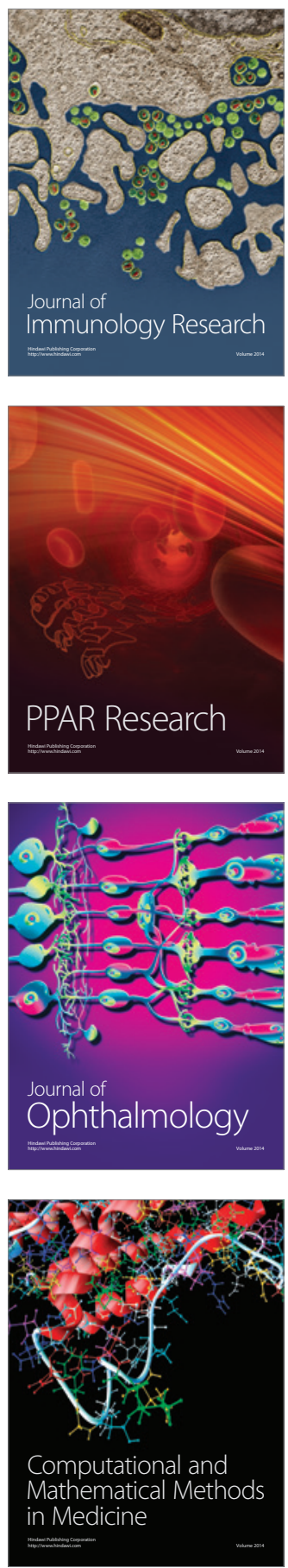

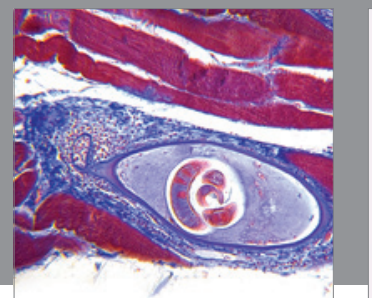

Gastroenterology

Research and Practice
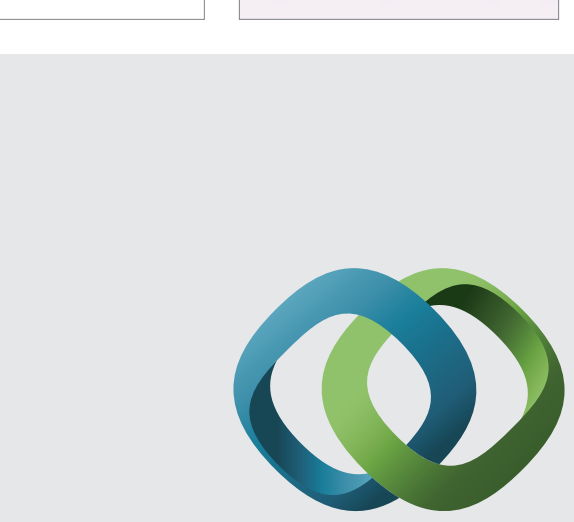

\section{Hindawi}

Submit your manuscripts at

http://www.hindawi.com
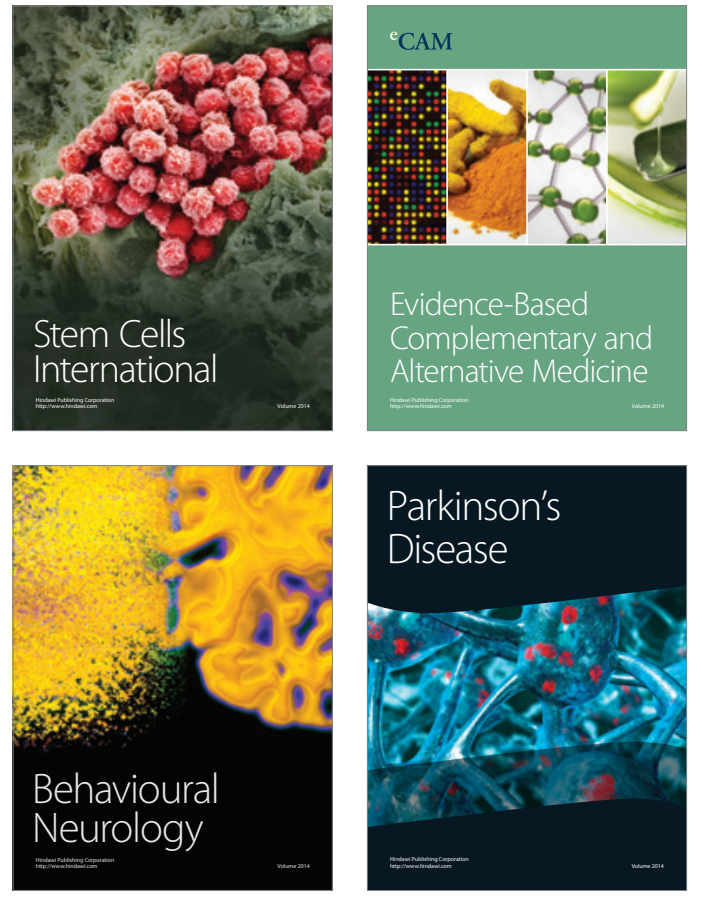
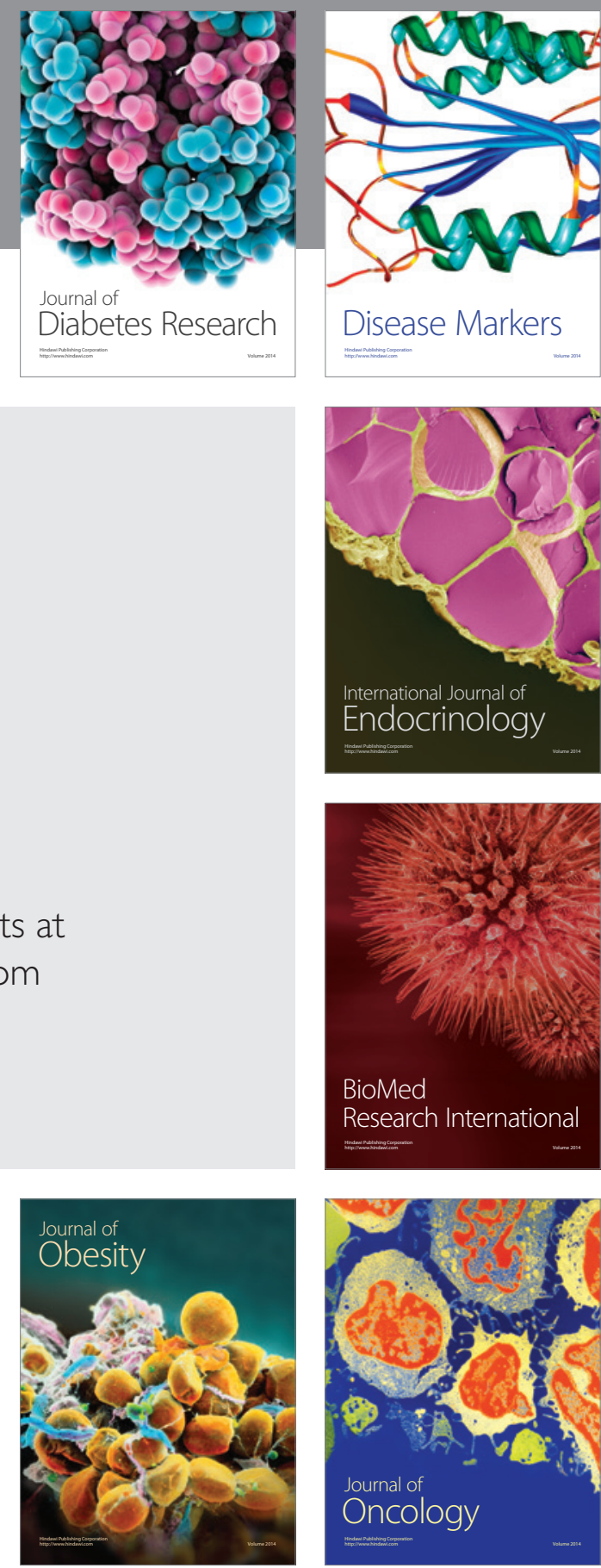

Disease Markers
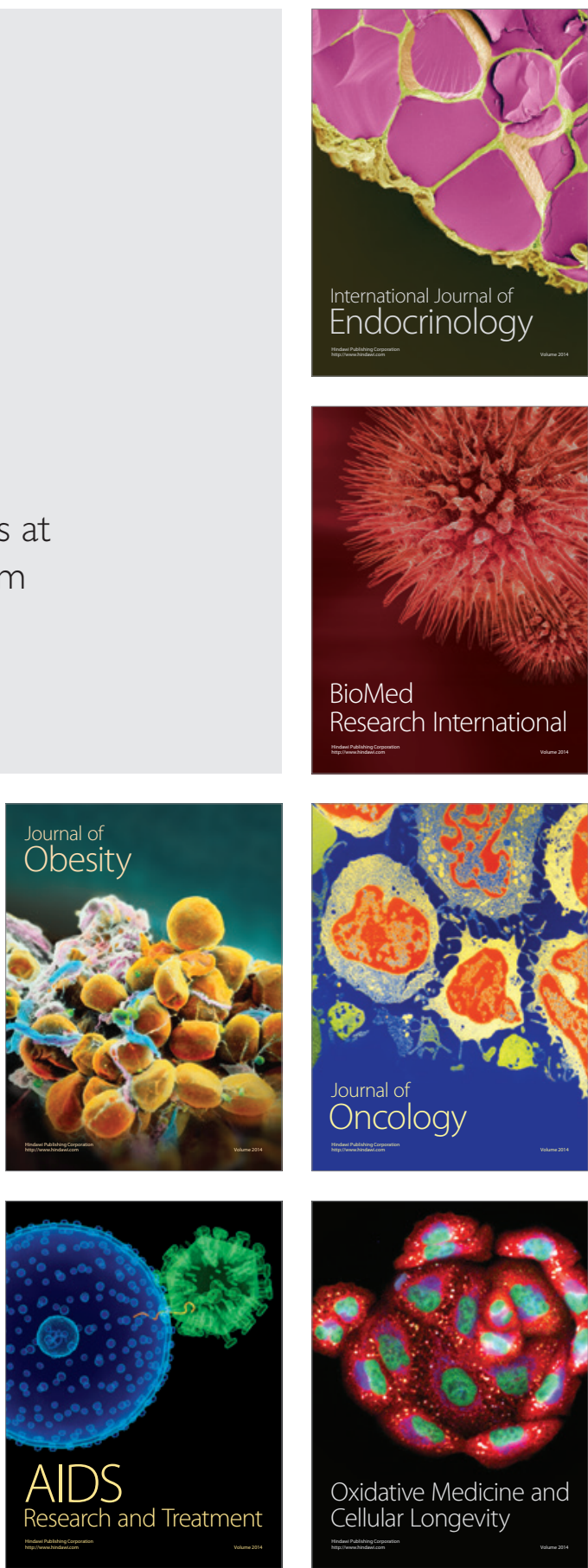